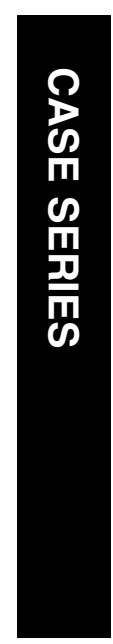

\section{Tuberculosis: an under-diagnosed aetiological agent in uveitis with an effective treatment}

\begin{abstract}
Purpose To highlight the diversity of clinical presentations with tubercular uveitis in a nonendemic setting, and discuss the diagnostic approach and an effective treatment.

Method Descriptive case series.

Results A total of 12 cases of varied presentations of tubercular uveitis diagnosed over a period of 1 year of which six cases are described in detail. Presentations included choroidal tuberculomas, multifocal choroiditis, recurrent granulomatous uveitis, panuveitis with cystoid macular oedema, and serpiginous choroiditis. All cases had a chronic or recurrent course and responded very well to antitubercular treatment. Diagnosis was mainly assisted by positive tuberculin testing.

Conclusion A high index of suspicion helps diagnose ocular tuberculosis in areas of low prevalence of the disease. It forms part of the differential diagnosis of any chronic or recurrent uveitis, especially in an at-risk patient. Antitubercular treatment seems highly effective.

Eye (2006) 20, 1068-1073. doi:10.1038/sj.eye.6702093; published online 7 October 2005
\end{abstract}

${ }^{1}$ Leeds General Infirmary, Leeds, West Yorkshire, UK

${ }^{2}$ Dewsbury and District Hospital, Leeds, West Yorkshire, UK

Correspondence: AR Reddy, Department of

Ophthalmology, Clarendon Wing Leeds

General Infirmary,

Leeds, West Yorkshire, LS2 9NS, UK

Tel: + 44113392 2704;

Fax +441132926239.

E-mail: arreddy@

rcsed.ac.uk

Received: 20 April 2005 Accepted in revised form: 3 August 2005

Published online: 7 October 2005
Keywords: uveitis; tuberculosis; diagnosis; tuberculin test; antitubercular therapy

\section{Introduction}

Tuberculosis (TB) is a communicable disease caused by Mycobacterium tuberculosis or related members of the TB complex. About $10 \%$ of infected individuals become symptomatic; $90 \%$ remain infected for the rest of their lives without manifesting the disease. ${ }^{1}$ Ocular TB is defined as an infection by $M$. tuberculosis in the eye,
D Varma', S Anand ${ }^{1}$, AR Reddy ${ }^{1}$, A Das ${ }^{1}$, JP Watson', DC Currie ${ }^{2}$, I Sutcliffe ${ }^{2}$ and OC Backhouse $\mathrm{B}^{1,2}$ around the eye, or on its surface. Classically, ocular TB has been divided into two types: primary and secondary. Primary disease implies that the eye is the initial port of entry while in secondary disease the organisms spread to the eye haematogenously. Primary disease includes conjunctival, corneal, and scleral disease, while tuberculous uveitis is a manifestation of secondary disease. The most common clinical findings of intraocular TB include solitary or multiple choroidal nodules, choroiditis, and retinal vasculitis. ${ }^{2-5}$ The choroidal nodules suggest direct haematogenous infection while the vasculitis and choroiditis are more likely to be the result of immune hypersensitivity.

Anterior tuberculous uveitis is typically granulomatous and an accompanying vitritis is not uncommon. ${ }^{2}$ TB should be considered in the differential diagnosis of chronic anterior granulomatous uveitis with or without posterior segment involvement. The absence of clinically evident pulmonary TB does not rule out the possibility of ocular TB, as approximately $60 \%$ of patients with extra pulmonary TB have no evidence of pulmonary TB. ${ }^{6}$

We present a case series of 12 patients who presented with manifestations of ocular TB to a UK hospital eye unit over a period of 1 year of which six differing examples of the varied clinical manifestations are described.

\section{Methods}

This is a retrospective and descriptive case series. The two hospitals serve a 'catchment' population of around one million, about $10 \%$ being of Asian ethnic origin. All the patients described hailed from the catchment area of these two hospitals and were referred to the regional uveitis clinic at Leeds (run under the supervision of OCB). Chest physicians of respective hospitals were involved in 
administering and monitoring the anti-TB treatment (JPW, DCC, IS).

\section{Results}

Table 1 outlines the clinical presentation of all 12 cases seen over a 1-year period.

\section{Case 1}

A 49-year-old male of Asian origin with a past ocular history of recurrent uveitis for 15 years presented to the eye clinic with a complaint of progressive decrease in visual acuity (VA) in his left eye (LE) over 1 week. He emigrated from Pakistan to the UK in 1971. His last visit to Pakistan was 2 years prior to presentation. The patient had not received BCG vaccination. There was neither past medical history of TB nor a history of known contact with a TB patient. At presentation, the best-corrected VA was $6 / 9$ in the right eye (RE) and 6/36 in the LE. Ocular examination revealed a panuveitis and a choroidal tuberculoma under the papillo-macular bundle in the LE (Figure 1a). The RE was normal. He was commenced on high-dose systemic steroids (oral prednisolone, $100 \mathrm{mg}$ daily) due to the vision-threatening nature of the lesion and an opinion of the chest physician sought. The VA and clinical features showed only a marginal improvement over the next 2 weeks. Medical examination revealed a clear chest with no cervical lymphadenopathy. Sarcoidosis and TB were considered in the differential diagnosis. Routine blood tests including chest $\mathrm{X}$-ray, serum angiotensin converting enzyme assay (ACE), plasma viscosity, C-reactive protein, and liver function tests were normal. Heaf test was positive (grade IV) despite being on high-dose prednisolone, strongly supporting a diagnosis of ocular TB. The patient was commenced on a standard course of antitubercular treatment (ATT) (2 months of rifampicin, isoniazid, pyrazinamide, and ethambutol followed by 4 months of rifampicin and isoniazid) and a reducing oral steroid dose. She had been only on oral steroids for 3 weeks prior to starting ATT. A dramatic improvement in the uveitis and VA was noted over an 8-week period. Now 2 years after completing ATT, the eyes remain quiet off all treatment with VA of 6/6 RE and 6/5 LE.

\section{Case 2}

A 31-year-old male of Asian origin presented to the accident and emergency department with a 3-day history of painful RE and loss of temporal field of vision. He had recently been diagnosed with pulmonary $\mathrm{TB}$ and had been on ATT for 6 weeks. His presenting VA was 6/18 in the RE and 6/9 in the LE. There were $2+$ cells in the anterior chamber, vitritis, and a raised deep white choroidal tuberculoma nasal to the optic disc with

Table 1 The 12 cases treated over a 1-year period

\begin{tabular}{|c|c|c|c|c|c|c|}
\hline $\begin{array}{l}\text { Case } \\
\text { number }\end{array}$ & $\begin{array}{l}\text { Age } \\
\text { (years) }\end{array}$ & Sex & Race/ethnic origin & Clinical presentation & Tuberculin test & $\begin{array}{l}\text { Response } \\
\text { to ATT }\end{array}$ \\
\hline 1 & 51 & Male & Asian/Pakistani & $\begin{array}{l}\text { Panuveitis and Choroidal } \\
\text { Tuberculoma left eye }\end{array}$ & Heaf grade 4 & Excellent \\
\hline 2 & 31 & Male & Asian/Indian & $\begin{array}{l}\text { Vitritis, peripapillary choroidal } \\
\text { tuberculoma }\end{array}$ & $\begin{array}{l}\text { Not performed (recent } \\
\text { diagnosis of pulmonary TB) }\end{array}$ & Excellent \\
\hline 3 & 71 & Female & Asian/Pakistani & $\begin{array}{l}\text { Bilateral recurrent anterior } \\
\text { granulomatous uveitis }\end{array}$ & Mantoux $20 \mathrm{~mm}$ induration & Excellent \\
\hline 4 & 34 & Male & Asian/Pakistani & $\begin{array}{l}\text { Multifocal choroiditis, recurrent } \\
\text { nongranulomatous panuveitis }\end{array}$ & Mantoux $32 \mathrm{~mm}$ induration & Excellent \\
\hline 5 & 51 & Female & Asian/Pakistani & $\begin{array}{l}\text { Uveitis post-cataract surgery, later } \\
\text { bilateral panuveitis }\end{array}$ & Heaf grade III & Excellent \\
\hline 6 & 63 & Male & Caucasian & $\begin{array}{l}\text { Serpiginous Choroiditis, recurrent } \\
\text { panuveitis }\end{array}$ & Heaf grade IV & Excellent \\
\hline 7 & 49 & Male & Asian/Pakistani & Recurrent anterior Uveitis right eye & Heaf grade IV & Excellent \\
\hline 8 & 15 & Male & Asian/Pakistani & $\begin{array}{l}\text { Granulomatous panuveitis, } \\
\text { periphlebitis }\end{array}$ & Heaf grade IV & Excellent \\
\hline 9 & 59 & Male & Asian/Pakistani & Periphlebitis, vitreous haemorrhage & Mantoux $25 \mathrm{~mm}$ induration & Excellent \\
\hline 10 & 51 & Male & Asian/Pakistani & $\begin{array}{l}\text { Recurrent non-granulomatous } \\
\text { uveitis, snow balls in vitreous } \\
\text { right eye }\end{array}$ & Heaf grade IV & Excellent \\
\hline 11 & 47 & Male & Asian/Pakistani & $\begin{array}{l}\text { Bilateral recurrent intermediate } \\
\text { uveitis }\end{array}$ & Mantoux $18 \mathrm{~mm}$ induration & Moderate \\
\hline 12 & 60 & Male & Asian/Indian & Anterior uveitis & Mantoux $15 \mathrm{~mm}$ induration & Excellent \\
\hline
\end{tabular}

ATT: anti-TB treatment. 


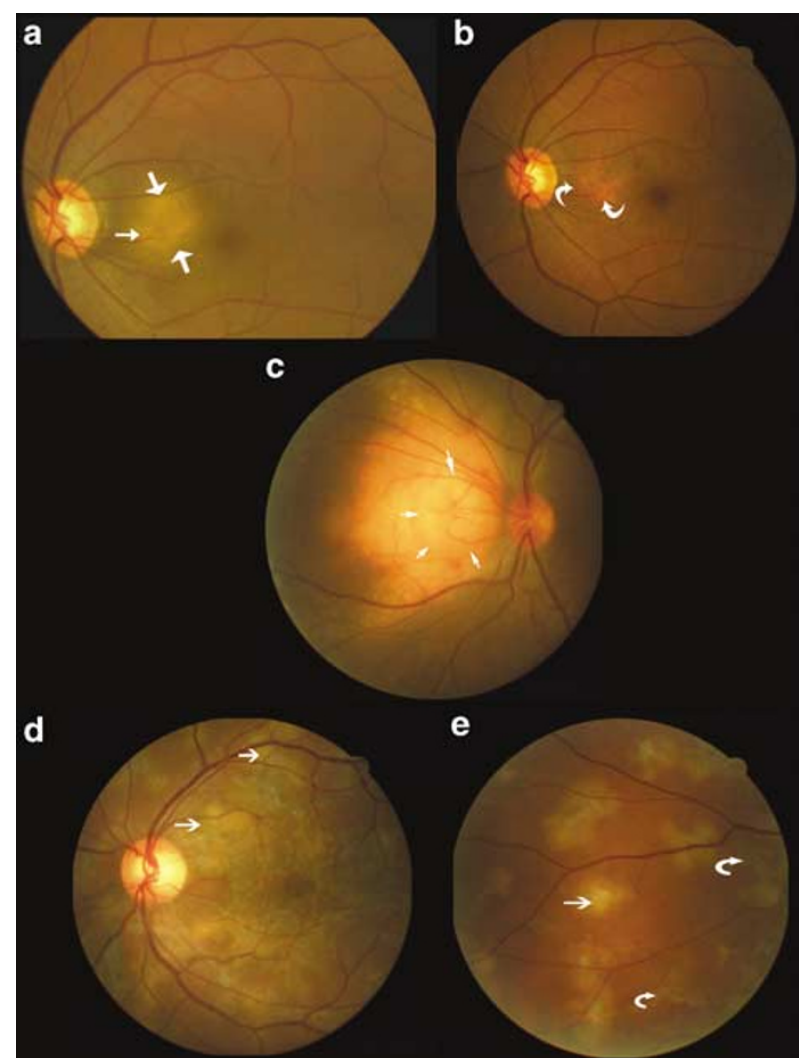

Figure $1 \quad(a, b)$ Case 1 showing large peripapillary choroiditis patch before and after ATT; (c) case 2 showing a very large choroidal tubercle; $(\mathrm{d}, \mathrm{e})$ case 4 showing multifocal choroiditis before and after ATT. (Straight arrows indicate active lesions and curved arrows healed lesions.)

associated blurring of the nasal disc margin and macular folds in the RE (Figure 1c). LE showed scattered chorioretinitis patches. Oral prednisolone ( $100 \mathrm{mg}$ daily) with calcium supplements and antacids were added to his anti-TB regime due to the sight-threatening nature of the lesions. After 1 week, the lesion had dramatically decreased in size, the VA had improved to $6 / 12$, and this improvement continued over the weeks following.

\section{Case 3}

A 61-year-old female of Asian origin presented to the eye clinic with a flare up of acute anterior granulomatous uveitis in the LE. She had been treated for bilateral recurrent nongranulomatous uveitis for 20 years with topical steroids and cycloplegics. Relevant past medical history included an episode of pyrexia associated with chest infection in 1998 for which she had been extensively investigated. She had not been treated with ATT because at that time her sputum culture for acid-fast bacilli was negative. The presenting VA was $6 / 36$ in the $\mathrm{RE}$ and 6/24 in the LE. There was evidence of acute anterior granulomatous uveitis in both eyes (Figure 1). Posterior segment examination was normal. A 1:1000 Mantoux test (10 TU purified protein derivative (ppd)) was strongly positive at $20 \mathrm{~mm}$. She was commenced on standard ATT on the advice of the chest physician. The uveitis resolved completely within 6 weeks of starting ATT and has not recurred for more than 1 year since.

\section{Case 4}

A 32-year-old Asian male presented with blurred vision in both eyes of 3 weeks duration associated with pain and long-standing floaters that had become more noticeable over the preceding fortnight. He had emigrated from Pakistan to the UK 3 years earlier. He had not received BCG immunization. There was no known history of TB or known contact with a TB patient. VA was $6 / 9$ in the RE and 6/18 in the LE. Examination revealed bilateral anterior uveitis with a few fine keratic precipitates, bilateral vitritis, and bilateral active choroiditis as well as old choroiditis scars (Figure 1d, e). Systemic investigations including full blood count (FBC), erythrocyte sedimentation rate (ESR), serum ACE, and chest $\mathrm{X}$-ray were normal. Antinuclear antibodies were weakly positive. Lyme and toxoplasma titre were negative. Mantoux testing (1:1000) was strongly positive at $32 \mathrm{~mm}$. The patient was commenced on standard ATT and oral prednisolone $(60 \mathrm{mg} /$ day). The ocular findings improved over the following weeks and the steroids were tapered and withdrawn. At 2 months after presentation, the VA in both eyes was 6/9 and the lesions appeared inactive with signs of pigmentation. Now 14 months after completing ATT there has been no recurrence of ocular inflammation.

\section{Case 5}

A 51-year-old Asian female underwent uncomplicated right cataract surgery. Postoperatively, she developed a low-grade chronic anterior uveitis with cystoid macular oedema in the operated eye. This was controlled on topical steroids with a VA of 6/9 until she presented with a flare up 7 months later. VA had dropped to $6 / 24$ in the $\mathrm{RE}$ and $6 / 18$ in the LE (previously 6/6). There was bilateral vitritis with marked cystoid macular oedema in the RE. The patient was systemically well with no previous history of uveitis. She denied previous BCG vaccination, recent foreign travel, or recent contact with a known case of TB. There was a history of contact 20 years previously when her father had been treated for TB. A routine uveitis screen was carried out, which showed a slightly elevated serum ACE level of $70.5 \mathrm{IU} / \mathrm{ml}$ (reference range 8-52 IU $/ \mathrm{ml}$ ). Chest X-ray was normal. A provisional diagnosis of sarcoidosis was made. 
However, in view of the family history, a Heaf test was organized before commencing steroid treatment. This was positive at grade II-III. In consultation with the chest physicians, it was considered unsafe to commence steroids with this result, especially in the absence of previous BCG vaccination. The patient was started on standard ATT. A few weeks into treatment, oral steroids were added. The uveitis resolved rapidly with return of VA to 6/9 in each eye. The patient did well over a 6-month follow-up. There were no recurrences.

\section{Case 6}

A 63-year-old Caucasian male was referred to the uveitis clinic with possible serpiginous choroiditis. He had a 9-year history of bilateral chronic panuveitis with recurrent acute exacerbations in spite of being on maintenance topical steroids almost continuously during this period. There was a history of active left choroiditis at initial presentation with a scar under macula suggestive of 'serpiginous choroiditis' with visual acuities of 6/6 in the RE and 6/60 in the LE. Intraocular pressures were controlled on Timolol having previously been raised due to steroid drops. At the time of referral, he had a 1-month history of further flare up of right panuveitis. Anterior chamber reaction had settled on topical steroid but vitritis persisted decreasing the right VA to $6 / 12$. He had been treated with prednisolone $40 \mathrm{mg}$ tapered over 6 weeks but had no significant improvement. Systemic investigations including FBC, ESR, serum ACE, chest X-ray, antinuclear antibodies, and VDRL were normal but Heaf test was positive (grade IV). On standard ATT, the vitritis cleared and the vision improved to $6 / 6$ in the RE. After 9 months, after finishing ATT, both eyes remain quiescent on no medication.

\section{Discussion}

In recent years, there has been a renewed interest in $\mathrm{TB}$ stimulated by a rising incidence, the spread of the HIV pandemic and the emergence of multidrug-resistant strains. ${ }^{7}$ In England and Wales, the annual rate of TB in 1998 was 10.93 per 100000 population, an increase of $11 \%$ since 1993 and $21 \%$ since $1988 .^{8}$ By 2002, it had risen a further $18 \%$ to 12.9 per $100000 .^{9}$ The major factor accounting for this increase is immigration from countries with high levels of endemic disease, although HIV coinfection has also contributed. ${ }^{8}$ Ethnic origin (south Asian) and recent travel to an endemic area were important risk factors in our series of cases. Poor socioeconomic conditions, immunosuppression, and general debility continue to be important predisposing factors irrespective of ethnic origin and should not be overlooked. ${ }^{10-12}$
Ocular TB presents a complex clinical problem due to a wide spectrum of presentations and difficulty in diagnosis. ${ }^{5,6,7,13}$ Uveitis is the most common ocular manifestation and can present as anterior, posterior, or panuveitis. ${ }^{1}$ Posterior segment features most classically associated with tuberculous uveitis ${ }^{1}$ include disseminated choroiditis as seen in case 5 and choroidal tubercle or focal choroiditis as seen in cases 1 and 2 . Tubercular anterior uveitis is typically granulomatous and may occur with or without posterior segment involvement, ${ }^{1}$ as in case 3 . In typical presentations such as these, TB is usually included in the differential diagnosis but the challenge is to recognize that atypical manifestations are common and can only be picked up with an index of suspicion. This is especially important, as TB is one of the few causes of uveitis with a definite effective treatment and where standard treatment with steroids in the absence of ATT could be sight or life threatening. Case 6 is notable in this regard and typifies the dilemma between TB and sarcoid. The presentation in this case was in a postoperative setting and with nonspecific panuveitis and cystoid macular oedema, none of which fit classically with TB. However, in view of the ethnic origin and family history, it was included in the differential diagnosis. The remarkable response to treatment, with rapid improvement in VA on ATT alone, confirmed the diagnosis. The spectrum of ocular TB is still evolving. Gupta et $a l^{14}$ recently reported a series of seven cases with a new morphological presentation in the form of serpiginous-like choroiditis. All seven cases had fundus features suggestive of this condition but continued to progress in spite of adequate steroid treatment and subsequently responded to ATT. Case 6 in our series had a similar clinical course. These cases highlight the importance of considering TB in any kind of uveitis presentation in an at-risk patient, especially if the clinical course is chronic or recurrent.

The diagnosis of ocular TB is frequently presumptive. ${ }^{7}$ Definite diagnosis relies on demonstration of tubercle bacilli in tissue but this is fraught with the difficulty of obtaining ocular tissue for biopsy in seeing eyes due to the invasive nature of the procedure. Ocular manifestations may result from a delayed hypersensitivity reaction in the absence of any infectious agent ${ }^{7}$ making the analysis of a fluid sample from the eye less sensitive. The other approach is to evaluate for systemic evidence of the disease in a patient with suggestive ocular features, but the absence of clinically evident systemic TB does not rule out ocular TB. ${ }^{1}$ Histopathologically proven intraocular TB has been shown to occur in patients with no signs or symptoms on TB testing other than a reactive skin test. ${ }^{1}$ Negative chest $X$-ray and sputum testing have limited exclusion value, as $81 \%$ of patients with extrapulmonary TB in England 
and Wales have no evidence of pulmonary disease. ${ }^{8}$ Recently, polymerase chain reaction has been used for detecting tubercular DNA in ocular fluid samples. ${ }^{15-17}$ While the initial results are promising, ${ }^{18}$ doubts have been expressed about its utility, as many ocular manifestations may represent a delayed immune hypersensitivity reaction rather than a direct infection. ${ }^{19}$ In other sites, molecular techniques have good specificity, but sensitivity is only around $77 \% .^{20}$ Further development and standardization of these techniques is needed to clarify their scope. At the present state of evidence, the authors do not feel justified in performing an invasive procedure to obtain ocular tissue or fluid samples for molecular testing in a patient with a clinical diagnosis of ocular TB.

Thus, the widely accepted approach to the diagnosis of ocular TB is based on clinical findings consistent with TB, positive tuberculin skin testing and absence of any other systemic disease to account for the uveitis. ${ }^{7,19}$ Tuberculin testing can be in the form of a Mantoux or a Heaf test, and the results need to be interpreted in light of previous BCG vaccination, ${ }^{21}$ exposure to nontuberculous mycobacteria, ${ }^{22}$ and any systemic immunosuppression. ${ }^{7}$ Interpretation of tuberculin skin testing can be difficult. There is no specific amount of induration that confirms $\mathrm{TB}$, nor does a negative test exclude TB. ${ }^{7}$ In the United Kingdom, a Heaf test is regarded as positive at grade II or more in subjects without prior BCG, or grade III or more in those with prior BCG. A Mantoux test response of more than $15 \mathrm{~mm}$ of induration in response to $10 \mathrm{TU}$ ppd is regarded as positive. ${ }^{23}$ Guidelines for interpreting Mantoux tests will vary in different countries where different strengths are used. A positive test, assuming it is not due to BCG or to exposure to nontuberculous mycobacteria, indicates infection with M. tuberculosis, but does not distinguish between latent TB infection and active disease. The disease may be associated with weak or negative reaction, especially in older people, the malnourished, and the

immunosuppressed. ${ }^{7}$ Some patients are intrinsically poor reactors. ${ }^{7}$ False-negative results as high as $17^{7}$ and $29 \%{ }^{24}$ have been reported.

Newer tests are being developed based on gamma interferon production by $\mathrm{T}$ cells sensitized to specific antigens, which are specific to $M$. tuberculosis and therefore not influenced by BCG or most nontuberculous bacteria. These tests, including Quantiferon TB Gold ${ }^{25}$ and enzyme-linked immunospot (ELISPOT) test, ${ }^{26}$ may prove useful in the future.

All our patients except case 2 had positive skin testing as the only supporting evidence of ocular TB. Latent TB infection is not uncommon in people of Asian origin in the United Kingdom, although this group is also at higher risk of the disease. ${ }^{8}$ The skin test alone cannot confirm the diagnosis of TB. However, the subsequent excellent response to ATT confirmed the diagnosis.

It might be questioned whether the clinical responses, we observed were due to ATT, or simply a response to the steroids that were also prescribed. However, in three of our cases (cases 1, 3, and 6), there had been persistent or recurrent uveitis over many years, despite previous courses of steroid treatment. Only after commencement of ATT, was there a sustained clinical response with no further relapses. While it might be desirable diagnostically to delay steroid treatment in order to assess the response to ATT, this must be balanced with the risk of loss of sight. The use of steroids at the same time as ATT is recommended in other situations where inflammation and fibrosis caused by TB may lead to long-term complications, for example, tuberculous meningitis or pericarditis. ${ }^{27}$

As a result of the difficulties with definitive diagnosis, a multidisciplinary approach involving a physician with a special interest in TB, from an early stage, is useful.

In summary, ocular TB is a treatable cause of uveitis. There is a wide spectrum of presentations and a high index of suspicion is needed, especially in at-risk patients for timely diagnosis and treatment. Further, the dramatic response to ATT in our case series may justify empirical treatment based on a high index of clinical suspicion and positive tuberculin test.

\section{Competing interests}

None of the authors have any financial interests in any of the products or equipment mentioned in the article.

\section{References}

1 Samson MC, Foster CS. Tuberculosis. In: Foster CS, Vitale AT (eds). Diagnosis and Treatment of Uveitis. WB Saunders Company: Philadelphia, 2002, pp 264-272.

2 Rosen PH, Spalton DJ, Graham EM. Intraocular tuberculosis. Eye 1990; 4: 486-492.

3 Ishihara M, Ohno S. [Ocular tuberculosis]. Nippon Rinsho 1998; 56: 3157-3161.

4 Cangemi FE, Friedman AH, Josephberg R. Tuberculoma of the choroid. Ophthalmology 1980; 87: 252-258.

5 Biswas J, Madhavan HN, Gopal L, Badrinath SS. Intraocular tuberculosis. Clinicopathologic study of five cases. Retina 1995; 15: 461-468 Review.

6 Alvarez S, McCabe WR. Extrapulmonary tuberculosis revisited: a review of experience at Boston City and other hospitals. (Baltimore) 1984; 63: 25-55.

7 Bodaghi B, LeHoang P. Ocular tuberculosis [review]. Curr Opin Ophthalmol 2000; 11: 443-448.

8 Rose AM, Watson JM, Graham C, Nunn AJ, Drobniewski F, Ormerod LP et al. Tuberculosis at the end of 20th century in England and Wales: results of a national survey in 1998. Thorax 2001; 56(3): 173-179.

9 www.hpa.org.uk/infections/topics_az/tb/pdf/. accessed 9/8/04. 
10 Rodger A, Jaffar S, Paynter S, Hayward A, Carless J, Maguire H. Delay in the diagnosis of pulmonary tuberculosis, London, 1998-2000: analysis of surveillance data. BMJ 2003; 326: 909-910.

11 Hawker JI, Bakshi S, Ali S, Farrington C. Ecological analysis of ethnic differences in relation between tuberculosis and poverty. BMJ 1999; 319: 1031-1034.

12 Milburn HJ, Heather J. Primary tuberculosis. Curr Opin Pulm Med 2001; 7: 133-141.

13 Sheu S, Shyu J, Chen LM, Chen YY, Chirn SC, Wang JS. Ocular manifestations of tuberculosis. Ophthalmology 2001; 108: $1580-1585$

14 Gupta V, Gupta A, Arora S, Bambery P, Dogra M, Agarwal A. Presumed tubercular serpiginouslike choroiditis. Clinical presentations and management. Ophthalmology 2003; 110: 1744-1749.

15 Kotake S, Kimura K, Yoshikawa K, Sasomoto Y, Matsuda A Nishikawa $T$ et al. Polymerase chain reaction for the detection of Mycobacterium tuberculosis in ocular tuberculosis [letter]. Am J Ophthal 1994; 117: 805-806.

16 Gupta V, Arora A, Gupta A, Ram J, Bambery P, Sehgal S. Management of presumed intraocular tuberculosis: possible role of polymerase chain reaction. Acta Ophthalmol Scand 1998; 76: 679-682.

17 Bowyer JD, Gormley R, Seth R, Downes RN, Lowe J. Choroidal tuberculosis diagnosed by polymerase chain reaction. A clinicopathologic case report. Ophthalmology 1999; 106: 290-294.

18 Ortega-Larrocea G, Bobadilla-del-Valle M, Ponce-de-Leon A, Sifuentes-Osornio J. Nested Polymerase Chain reaction for Mycobacterium tuberculosis DNA detection in aqueous and vitreous of patients with uveitis. Arch Med Res 2003; 34: 116-119.

19 Morimura Y, Okada A, Kawahara S. Tuberculin skin testing in uveitis patients and treatment of presumed intraocular tuberculosis in Japan. Ophthalmology 2002; 109 851-857.

20 Lim TK, Mukhopadhyay A, Gough A, Khoo-Kay L, Khoo-See M, Lee Kang $\mathrm{H}$ et al. Role of clinical judgement in the application of a nucleic acid amplification test for the rapid diagnosis of pulmonary tuberculosis. Chest 2003; 124: $902-908$

21 Wang L, Turner MO, Elwood RK, Schulzer M Fitzgerald JM. A meta-analysis of the effect of Bacille Calmette Guerin vaccination on tuberculin skin test measurements. Thorax 2002; 57: 804-809.

22 Bruins J, Gribnau JH, Bwire R. Investigation into typical and atypical tuberculin sensitivity in the Royal Netherlands Army, resulting in a more rational indication for isoniazid prophylaxis. Tuberc Lung Dis 1995; 76: 540-544.

23 Joint Tuberculosis Committee of the British Thoracic Society. Control and prevention of tuberculosis in the United Kingdom: code of Practice 2000. Thorax 2000; 55: 887-901.

24 Singh SD, Williams AJ, Vargo DL, Woodcock A. An investigation of the prevalence of tuberculin positive reactors amongst healthy subjects in Cheshire. Thorax 1999; 54(Suppl 3): 49.

25 Moru T, Sakatani M, Yamagishi F, Takashima T, Kawabe Y, Nagao $\mathrm{K}$ et al. Specific detection of tuberculosis infection: an interferon- $\gamma$-based assay using new antigens. Am J Respir Crit Care Med 2004; 170: 59-64.

26 Ewer K, Deeks J, Alvarez L, Bryant G, Waller S, Andersen P et al. Comparison of T-cell-based assay with tuberculin skin test for diagnosis of Mycobacterium tuberculosis infection in a school tuberculosis outbreak. Lancet 2003; 361: 1168-1173.

27 Joint Tuberculosis Committee of the British Thoracic Society. Chemotherapy and management of tuberculosis in the United Kingdom: recommendations 1998. Thorax 1998; 53 536-548. 